RECYT

Año 21 / № 32 / 2019 / 71-75

\title{
Modelado matemático del secado de fideos libres de gluten, influencia de la velocidad del aire
}

\section{Mathematical modeling of gluten-free noodles drying, influence of air velocity}

\author{
Paola S. Chigal'1 , Laura B. Milde², Luis A. Brumovsky² \\ 1- Consejo Nacional de Investigaciones Científicas y Técnicas (CONICET). Posadas, Argentina. \\ 2- Departamento de Ciencia y Tecnología de los Alimentos. Facultad de Ciencias Exactas, Químicas \\ y Naturales. Universidad Nacional de Misiones (UNaM). Misiones, Argentina. \\ *E-mail: paolachigal@gmail.com
}

\section{Resumen}

Se modeló matemáticamente la cinética de secado de pastas libres de gluten, y se estudió la influencia de la velocidad del aire sobre datos experimentales. Los fideos espagueti fueron elaborados mezclando fécula de mandioca, harina de maíz, leche en polvo, huevo, margarina y goma xántica, con agregado de agua. Se secaron a $40^{\circ} \mathrm{C}$ y a tres velocidades de aire: 0,$4 ; 0,8$ y $1,5 \mathrm{~m} / \mathrm{s}$. Se observó una disminución del tiempo de secado a medida que aumenta la velocidad del aire. Los datos se ajustaron a tres modelos matemáticos semi-empiricos de capa fina, se seleccionó como mejor ajuste la ecuación de Page $\left(\mathrm{R}^{2}=99 \%\right)$ para las tres velocidades analizadas. EI parámetro $\mathrm{k}$ de la ecuación de Page presentó dependencia con la velocidad de aire mientras que el parámetro $\mathrm{n}$ se consideró constante. Los resultados obtenidos podrían ser utilizados para la predicción del tiempo de secado de las pastas analizadas.

Palabras clave: Secado; Fideos libres de gluten; Modelado matemático; Ecuaciones de capa fina.

\section{Abstract}

The drying kinetics of gluten-free pasta was modeled mathematically, and the influence of air velocity on experimental data was studied. Spaghetti noodles were made by mixing cassava starch, corn flour, milk powder, egg, margarine and xanthan gum, with water added. They were dried at $40^{\circ} \mathrm{C}$ and three air velocities: $0.4 ; 0.8$ and $1.5 \mathrm{~m} / \mathrm{s}$. A decrease in drying times as the air velocity increases was observed. The data were adjusted to three semi-empirical mathematical models of thin layer; for the three velocities analyzed, the Page's equation was selected as the best fit. The parameter $k$ of Page's equation presented dependence with the air velocity while the parameter $\mathrm{n}$ was considered constant. The results obtained could be used to predict the drying time of the pastas analyzed.

Keywords: Drying; Gluten free noodles; Mathematical modeling; Thin layer equations.

\section{Introducción}

En muchos países, las pastas constituyen un alimento básico en la dieta de las personas [1], motivo por el cual la industria se encuentra en constante búsqueda de técnicas innovadoras principalmente en cuanto al proceso de deshidratación de las mismas. El secado se define como una operación en la que se produce la remoción de agua en pequeñas cantidades de un material por acción del calor. En los alimentos, su uso está orientado principalmente a la preservación de los mismos, debido a que los microorganismos que provocan la descomposición de los alimentos, no pueden crecer y multiplicarse en ausencia de agua; además, las enzimas que causan alteraciones en los alimentos, no pueden funcionar sin agua [2]. Todo esto hace que la vida útil del producto aumente significativamente.
El Código Alimentario Argentino define a los fideos secos como aquellos que han sufrido un proceso de desecación y exige que contengan una humedad no superior al 14\% $\mathrm{p} / \mathrm{p}[3]$.

El proceso de secado se lleva a cabo generalmente mediante convección forzada de aire caliente, por lo que la rapidez del proceso, y por lo tanto la cinética de secado, dependen tanto de factores del alimento (densidad, porosidad, composición, contenido de humedad inicial, geometría), como de factores operativos que incluyen la temperatura, la humedad y la velocidad de circulación del aire $[4,5$, $6,7]$. Estas variables operativas deben ser estrictamente controladas, de manera tal que la eliminación de agua sea lenta para evitar que el fideo se seque rápidamente y se endurezca antes la superficie que el interior, provocando la rotura del producto durante la manipulación [8]. Tal 
resultado tendría un impacto negativo en la aceptación del consumidor a causa de un mal aspecto y una calidad inferior de cocción [9]. El secado de la pasta en fábricas se realiza bajo condiciones variadas tanto de temperaturas (entre $\operatorname{los} 40^{\circ} \mathrm{C}$ y $105^{\circ} \mathrm{C}$ ) como de humedades relativas (entre el 40\% y el 95\%) (10). Estas condiciones deben estar relacionadas con las propiedades de la pasta como una función del tiempo, para determinar las variables operativas óptimas del proceso de acuerdo con cualquier criterio de calidad de la pasta [11].

El secado es una operación difícil de describir, debido a la complejidad de los fenómenos internos y externos que ocurren durante el mismo. Por ello se emplean modelos matemáticos que representen la cinética de secado de los alimentos y contribuyan a predecir tiempos de procesamiento y así optimizar la eficiencia del secado. Actualmente en la literatura se encuentran varios métodos propuestos para analizar el secado de productos alimenticios: modelos teóricos, semiempíricos y empíricos.

Dentro de los más usados se destacan los modelos de cinética de secado que se basan en relaciones semiempíricas de capa fina, que realizan una simplificación de las ecuaciones que describen el proceso [12]. Se basan en la suposición de que la disminución de la humedad es proporcional a la diferencia instantánea entre el contenido de humedad del material (asumida uniforme dentro del alimento) y el contenido de humedad en equilibrio con el aire de secado [13]. Entre las ecuaciones más utilizadas en la literatura para describir este modelo se encuentran las logarítmicas, la ecuación de Newton, de Page, de Henderson y Pabis, entre otras.

El objetivo del presente trabajo fue modelar matemáticamente la cinética de secado de pastas libres de gluten, y estudiar la influencia de la velocidad del aire sobre los datos experimentales.

\section{Materiales y métodos}

\section{Equipos de secado}

Las pruebas de secado se llevaron a cabo en la Planta piloto del módulo de ingeniería de la Facultad de Ciencias Exactas, Química y Naturales, ubicado en el Campus de la Universidad Nacional de Misiones. La temperatura aplicada para llevar a cabo los ensayos fue $40^{\circ} \mathrm{C}$, la misma se seleccionó porque, al tratarse de una pasta elaborada con harinas diferentes al trigo, su estructura es más frágil, presenta menor cantidad de agua en su formulación y el secado se produce en tiempos menores a los de las pastas tradicionales. Si se utilizaran temperaturas superiores, el secado se llevaría a cabo a velocidades tan elevadas que podría producirse sequedad excesiva en la superficie de la pasta volviéndola muy quebradiza y de baja calidad [7]. En cuanto a la humedad relativa del aire de secado, los equipos utilizados no cuentan con un instrumento capaz de regularla, por lo que la misma únicamente fue controlada mediante el uso de un termómetro con un algodón humedecido para medir la temperatura de bulbo húmedo y calcular la humedad relativa. En todas las determinaciones, los valores estuvieron en un rango de 60 a $65 \%$.

Para alcanzar las velocidades de aire en estudio, se utilizaron dos equipos de secado: un secadero de escala de laboratorio, con el cual se estudiaron las velocidades de 0,8 y $1,5 \mathrm{~m} / \mathrm{s}$, y un horno de secado por convección de aire (marca Dalvo, modelo EHRF, Argentina) para analizar valores de velocidades de aire de $0,4 \mathrm{~m} / \mathrm{s}$. El secadero de escala laboratorio consta de: una cámara de secado donde fueron colocadas las bandejas metálicas que contenían las muestras; un sistema de calentamiento del aire con resistencias eléctricas y un ventilador para la circulación del aire. Previo a las experiencias de secado, se acondicionó el funcionamiento del equipo durante 30 minutos, hasta operación estable $\left(40^{\circ} \mathrm{C}\right.$, y la velocidad correspondiente).

\section{Preparación de las muestras}

Se procedió a la elaboración de las pastas de manera artesanal, partiendo de una formulación compuesta por: fécula de mandioca (Montecarlo, Misiones) y harina de maíz (Indelma, Santa Fe) en proporción 80:20, 5\% de leche en polvo (Ilolay, Santa Fe), 6\% de materia grasa (Margadán, Buenos Aires), 12\% de huevo entero y 0,6\% de sal (Dos Anclas, San Luis), se incorporó 0,6\% de goma xántica (Parafarm, Buenos Aires), con la intención de reemplazar el gluten y proporcionar a las masas, elasticidad y mayor resistencia. Se agregó agua hasta formar una masa homogénea $\mathrm{y}$, mediante el uso de una máquina para elaborar pastas (Pluselectric, China), fue laminada entre dos rodillos lisos de acero inoxidable hasta un espesor de $2 \mathrm{~mm}$ y cortada entre dos rodillos acanalados para formar tiras de $1 \mathrm{~mm}$ de ancho, tipo espagueti. Se estandarizó el largo de las tiras en $20 \mathrm{~cm}$.

\section{Cinética del secado}

Para cada experiencia de secado, $50 \mathrm{~g}$ de pastas fueron colocadas en bandejas rectangulares de aluminio, extendidas y separadas para evitar que se peguen entre sí durante la operación. Las bandejas se pesaron inicialmente cada 10 minutos durante la primer hora de secado y luego cada 20 minutos hasta peso constante, de manera tal de evaluar la pérdida de agua y poder realizar los gráficos correspondientes (cinética y rapidez de secado). Las determinaciones se realizaron por duplicado y la humedad inicial de las muestras fue evaluada mediante la técnica $\mathrm{N}^{\circ} 925.09 \mathrm{de}$ la AOAC [14]. 


\section{Modelado matemático}

Se utilizaron tres modelos matemáticos de capa fina de los más utilizados en la literatura, para representar la cinética de secado experimental de pastas. En estos modelos, la variable dependiente es XR la cual representa la relación de humedad:

$X R=\frac{X t(b . s .)}{X 0(b . s .)}$

Donde: Xt es la humedad a un tiempo t y X0 es la humedad inicial, ambas en base seca. La Tabla 1 muestra las expresiones de los modelos seleccionados. Los valores de $\mathrm{k}, \mathrm{n}$ y a son parámetros de los modelos cinéticos.

Tabla 1: Ecuaciones de los modelos de ajuste de la cinética del secado

\begin{tabular}{|c|c|}
\hline Modelos & Ecuación \\
\hline Page & $\mathrm{XR}=\mathrm{e}^{\left(-\mathrm{k} \mathrm{t}^{\mathrm{n}}\right)} \quad$ (EC. 2) \\
\hline Newton & $\mathrm{XR}=\mathrm{e}^{(-\mathrm{k} \mathrm{t})} \quad$ (EC. 3) \\
\hline Henderson - Pabis & $\mathrm{XR}=\mathrm{a}^{(-\mathrm{k} \mathrm{t})} \quad$ (EC. 4) \\
\hline
\end{tabular}

\section{Análisis estadístico}

Los mejores ajustes serán aquellos que presenten valores más altos del estadístico $\mathrm{R}^{2}$, menores valores de Error Absoluto Medio (MAE) y Cuadrado del Error Medio (CME). Estos estadísticos fueron calculados con el software "Statgraphics plus 5.1”, mediante un análisis de Regresión no lineal. Las curvas, tanto experimentales como las modeladas fueron graficadas mediante el programa Microsoft Excel 2010.

\section{Resultados y Discusión}

\section{Cinética del secado}

La humedad inicial de las pastas fue de 55,8\% (b.s). Todas las curvas presentaron una forma exponencial, similar a las observadas en general para los productos alimenticios [2].

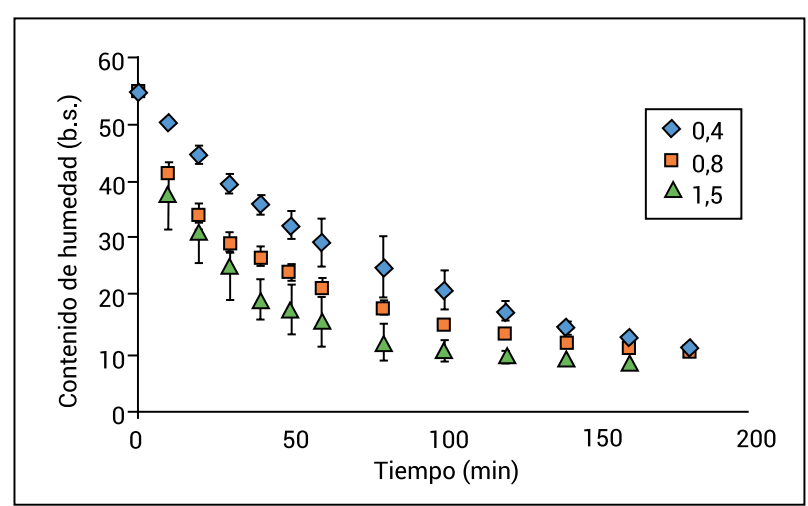

Figura 1: Curvas de secado de las pastas para velocidades de aire de 0,$4 ; 0,8$ y $1,5 \mathrm{~m} / \mathrm{s}$; valores medio y desvíos estándares.
En la Figura 1 se observa la influencia de la velocidad del aire sobre las curvas de secado de las muestras de fideos analizadas. Como era de esperarse, los tiempos de secado de las pastas variaron de manera inversamente proporcional con la velocidad del aire empleado, resultados similares a los obtenidos por Inazu, Iwasaki y Furuta (2003) [15] al evaluar la influencia de la velocidad del aire de secado de fideos tipo udon. Los tiempos hasta alcanzar una humedad menor a $14 \%$ fueron de: $160 \mathrm{~min}$ para la velocidad de 0,4 $\mathrm{m} / \mathrm{s}, 120 \mathrm{~min}$ para la velocidad de $0,8 \mathrm{~m} / \mathrm{s}$ y $60 \mathrm{~min}$ para la velocidad de $1,5 \mathrm{~m} / \mathrm{s}$. Puede decirse que la velocidad del aire influyó en la cinética de secado de las pastas incluso a la máxima velocidad estudiada, contrario a los resultados obtenidos por Andrieu y Stamatopoulos (1986) [16].

Las curvas de cinética de secado no presentaron un período inicial de ajuste; esto puede deberse a que la temperatura de secado es lo suficientemente baja y el material que ingresa a la cámara no necesita precalentamiento ya que alcanza la temperatura del aire rápidamente; este fenómeno es común en los productos alimenticios [17].

En la Figura 2 se presenta la gráfica con los valores medios y sus desvíos estándares de la rapidez de secado de las pastas en función del contenido de humedad, para cada una de las velocidades del aire de secado.

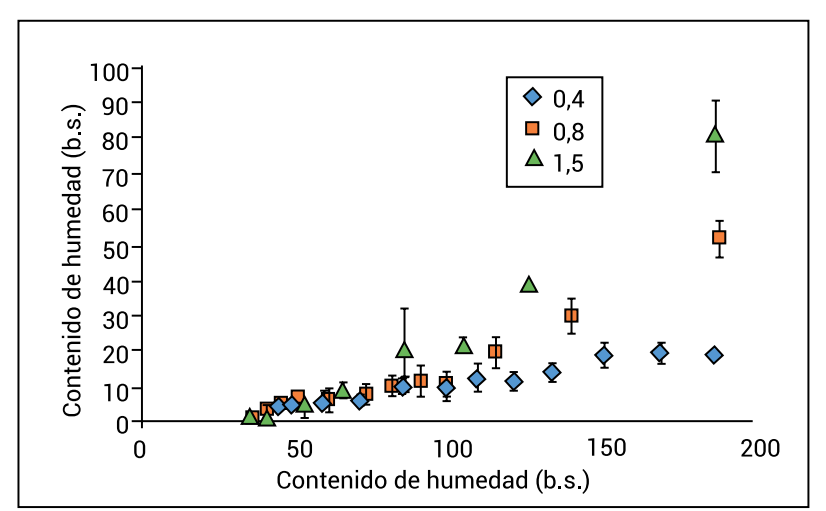

Figura 2: Curvas de rapidez de secado de las pastas para velocidades de aire de 0,$4 ; 0,8$ y 1,5 m/s; valores medios y desvíos estándares.

Se distingue en la Figura 2, que el secado se llevó a cabo en su mayor parte en el período de velocidad decreciente, no se evidenció el período de velocidad constante para ninguna de las experiencias de secado. Este fenómeno no resultó extraño considerando que no se observó humedad en la superficie de las pastas, al iniciar el proceso de secado. En las curvas se observa, principalmente para las velocidades de secado de 0,8 y $1,5 \mathrm{~m} / \mathrm{s}$, dos períodos de velocidad decreciente; solamente el primero con cierta tendencia a la linealidad. Resultados similares fueron obtenidos por otros autores cuando estudiaron la cinética de secado mediante convección forzada de aire caliente en distintos alimentos, como ser fideos elaborados con una mezcla de harinas diferentes al trigo $(18,19)$ o rodajas de batata [20]. 


\section{Modelos matemáticos de las curvas de secado}

A partir de los valores que se presentan en la Tabla 2 se puede observar que, excepto el modelo de Newton para la velocidad de aire de $1,5 \mathrm{~m} / \mathrm{s}$, los demás describieron satisfactoriamente las curvas de secado de las pastas analizadas. El modelo de Page es el que presentó los valores más altos del estadístico $\mathrm{R}^{2}$ y los menores valores para MAE y CME los que se mantuvieron constantes para las tres velocidades de aire evaluadas; por lo que se consideró como el modelo con mejor ajuste a los datos experimentales de cinética de secado (Figura 3).

Tabla 2: Resultados de los parámetros estadísticos y de ajuste de las ecuaciones de modelado cinético a distintas velocidades de aire.

\begin{tabular}{|c|c|c|c|c|}
\hline Modelos & Parámetros & $0,4 \mathrm{~m} / \mathrm{s}$ & $0,8 \mathrm{~m} / \mathrm{s}$ & $1,5 \mathrm{~m} / \mathrm{s}$ \\
\hline \multirow{5}{*}{ PAGE } & $\mathrm{R}^{2}(\%)$ & 99,28 & 99,81 & 99,48 \\
\hline & MAE & 0,0162 & 0,0072 & 0,0143 \\
\hline & CME & 0,0006 & 0,0001 & 0,0004 \\
\hline & K & 0,016 & 0,081 & 0,133 \\
\hline & $\mathrm{n}$ & 0,902 & 0,596 & 0,564 \\
\hline \multirow{4}{*}{ NEWTON } & $\mathrm{R}^{2}(\%)$ & 98,68 & 87,30 & 0 \\
\hline & MAE & 0,0222 & 0,0718 & 18,4 \\
\hline & CME & 0,0009 & 0,0074 & 640,9 \\
\hline & K & 0,009 & 0,015 & $\mathrm{k}<0$ \\
\hline \multirow{5}{*}{$\begin{array}{l}\text { HENDERSON- } \\
\text { PABIS }\end{array}$} & $\mathrm{R}^{2}(\%)$ & 98,83 & 92,84 & 91,81 \\
\hline & MAE & 0,0211 & 0,0287 & 0,0598 \\
\hline & CME & 0,0009 & 0,0047 & 0,0062 \\
\hline & a & 0,977 & 0,858 & 0,884 \\
\hline & K & 0,010 & 0,012 & 0,023 \\
\hline
\end{tabular}

Modelo $(0,4 \mathrm{~m} / \mathrm{s})$

Modelo $(0,8 \mathrm{~m} / \mathrm{s})$

Modelo $(1,5 \mathrm{~m} / \mathrm{s})$

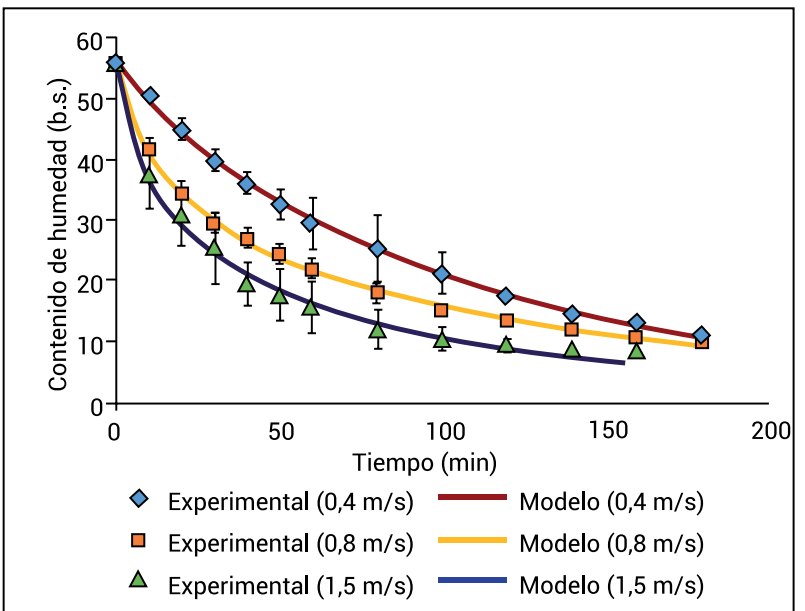

Figura 3: Curvas de secado (experimentales y modeladas mediante la ecuación de Page) para las pastas estudiadas.
El buen ajuste que presenta la ecuación de Page podría deberse a que, como las curvas de secado analizadas presentan tendencia exponencial, el parámetro $\mathrm{n}$ de dicha ecuación le otorgaría una mayor aproximación matemática sobre las curvas $(17,21$ y 22).

\section{Parámetros k y n de la ecuación de page}

Una vez seleccionada la ecuación de Page como la de mejor ajuste, se logró establecer una relación del tipo logarítmica entre el parámetro $\mathrm{k}$ y la velocidad del aire de secado, con un $\mathrm{R}^{2}$ de $99,8 \%$, obteniéndose una ecuación del tipo:

$\mathrm{k}=0,0889 \operatorname{In}(\mathrm{v}$ aire $)+0,0986$

Esta expresión muestra que el valor de k se incrementa a medida que aumenta la velocidad de aire. No se logró ajustar los valores del parámetro $\mathrm{n}$ a ningún modelo matemático, los $\mathrm{R}^{2}$ eran menores a $85 \%$ en todos los modelos analizados (lineal, logarítmico, exponencial y potencial); se optó por tomar un valor promedio entre las tres velocidades de aire y considerarlo constante $(0,687 \pm 0,169)$.

No se encontró bibliografía referente al análisis de los parámetros k y n de la ecuación de Page en función de la velocidad de aire en fideos, por lo que se recurrió a comparar con el efecto de los mismos en otros productos alimenticios; Sharma, Verma y Pathare (2005) [23] ajustaron el secado de rebanadas de cebollas mediante un secador infrarrojo convectivo al modelo de Page, observando que solo la constante $\mathrm{k}$ del modelo dependía de la temperatura y de la velocidad del aire de secado, mientras que el valor de $\mathrm{n}$ solo era dependiente de la temperatura y no de la velocidad de aire; coincidiendo con el presente trabajo.

\section{Conclusión}

A partir de la gráfica de cinética de secado, se concluyó que los fideos presentaron una curva del tipo exponencial, que depende de la velocidad del aire de secado. Los tiempos de secado de los fideos elaborados variaron de manera inversamente proporcional a la velocidad del aire de secado. De acuerdo a los resultados estadísticos obtenidos, el modelo de Page fue el que presentó el mejor ajuste de los datos experimentales, por lo que podría utilizarse como una buena herramienta para el cálculo estimado del tiempo de secado de los fideos libres de gluten analizados.

\section{Lista de abreviaturas}

$\mathrm{XR}=$ Relación de humedad

$\mathrm{Xt}$ (b.s.) = humedad a un tiempo $\mathrm{t}$ en base seca.

$\mathrm{X} 0$ (b.s.) $=$ humedad inicial en base seca 
k, n y a = parámetros o constantes de secado

$\mathrm{MAE}=$ Error Absoluto Medio

$\mathrm{CME}=$ Cuadrado del Error Medio

$\mathrm{v}$ aire $=$ velocidad del aire de secado

\section{Agradecimientos}

Los autores agradecen a la Universidad Nacional de Misiones (UNaM), el financiamiento del proyecto de investigación: "Fortificación de fideos secos a base de fécula de mandioca, con proteína de origen animal; características fisico-químicas y texturales". Al Dr. Santiago Holowaty por el apoyo técnico en el manejo de equipos y al alumno Donovan Rivero en la preparación de las muestras.

\section{Bibliografía}

1. Markowski, M.;Cenkowski, S.; Hatcher, D. W.; Dexter, J. E. y Edwards N. M. The effect of superheated-steam dehydrationkinetics on textural properties of asian noodles. American Society of Agricultural Engineers. 46(2): p. 389-395. 2003.

2. Geankoplis, C.J. Proceso de transporte y operaciones unitarias. Compañía Editorial Continental, S.A. de C.V., México. p. 579-609. 3era edición, 1998.

3. Código Alimentario Argentino (CAA). Capítulo IX (Alimentos Farináceos), Artículo 707. Actualización 2018.

4. Oti-Boateng, P. y Axtell, B. Técnicas de secado. Libro de consulta sobre tecnologías aplicadas al ciclo alimentario. Intermediate Technology Development Group. Peru. P. 70. 2da Edición. 1998.

5. Krokida, M. K.; Karathanos, v.; Maroulis, Z. y Kouris, D. M. Drying kinetics of some vegetables. Journal of Food Engineering. 59(4): p. 391-403. 2003.

6. Veladat, R.; Zokaee Ashtiani, F.; Rahmani, M. y Miri, т. Review of numerical modeling of pasta drying, a closer look into model parameters. Asia-Pacific Journal of Chemical Engineering, 7(2): p.159-170. 2012.

7. Larrosa, v.; Lorenzo, G.; Zaritzky, N. y Califano, A. Modelado matemático del secado de pastas libres de gluten en relación a la temperatura y humedad relativa del aire. Revista del laboratorio tecnológico del Uruguay (INNOTEC). 11: p. 54-58. 2016.

8. Johnston, K. w. y Dintheer, w. Pasta and semolina technology. Capítulo 6: Pasta drying. Editores: Kill, R. C. y Turnbull, K. p. 158-175. 2000.

9. Marchylo B. A. y Dexter J. E. Cereal Processing Techology. Capítulo: Pasta production. Editor: Owens G., 1ra Edición. Woodhead Publishing Limited, Inglaterra. 2001.

10. De Temmerman, J.; Verbovena, P.; Delcourb, J. A.; Nicolaïa, B. y Ramona, H. Drying model for cylindrical pasta shapes using desorption isotherms. Journal of Food Enginee- ring, 86(3), pp. 414-421. 2007.

11. Migliori, M.; Gabriele, D.: de Cindio, B. y Pollini, C. M. Modelling of high quality pasta drying: mathematical model and validation. Journal of Food Engineering, 69(4), pp. 387-397. 2005.

12. Collazo-Abreu, P. L.; Morejón-Mesa, Y.; Fernández-Chuairey, L. y Vázquez-Alfonso Y. Modelos matemáticos y experimentales para el análisis del secado solar de semillas. Revista Ciencias Técnicas Agropecuarias, 27(1), pp. 89-98. 2018.

13. Aversa, M.; Curcio, S.; Calabro, v. y Iorio, G. An analysis of the transport phenomena occurring during food drying process. Journal of Food Engineering, 78, pp. 922932. 2007.

14. Association of Official Analytical Chemists. Official Methods 925.09: Moisture. Air Oven Methods: Official Methods of Analysis of AOAC International, 2005.

15. Inazu, T.; Iwasaki, K. y Furuta, T. Effect of air velocityon fresh Japanese noodle (Udon) drying. Lebensm.-Wiss. U.-Technol. 36(2), pp. 277-280. 2003.

16. Andrieu, J. y Stamatopoulos, A. A. Durum Wheat Pasta Drying Kinetics. Lebensm-Wiss Technol, 19(6), 448456. 1986.

17. Azzouz, S.; Guizani, A.; Jomaa, w y Belghith, A. Moisture diffusivity and drying kinetic equation of convective drying of grapes. Journal of Food Engineering, 55(4), pp: 323-330. 2002.

18. Kaushal, P. y Sharma, н. к. Convective dehydration kinetics of noodles prepared from taro (Colocasia esculenta), rice (Oryza sativa) and pigeon pea (Cajanus cajan) flours. Agric Eng Int: CIGR Journal, 15 (4), pp. 202212. 2013.

19. Kongkiattisak, P. y Songsermpong, S. Effect of temperature and velocity of drying air on kinetics quality and energy consumption in drying process of rice noodles. Kasetsart J. (Nat. Sci.) 46, pp: 603 - 619. 2012

20. Diamante, L. M. y Munro, P. A. Mathematical modeling of hot air drying of sweet potato slices. International Journal of Food Science and Technology. 26(1), pp: 99-109. 1991.

21. Doymaz, I. Drying characteristics and kinetics of okra. Journal of Food Engineering 69(3), pp: 275-279. 2005.

22. Mwithiga, G. y Olwal, J. o. The drying kinetics of kale (Brassica oleracea) in a convective hot air dryer. Journal of Food Engineering, 71(4), pp: 373-378. 2005.

23. Sharma, G. P.; Verma; R. C. y Pathare, P. Mathematical modeling of infrared radiation thin layer drying of onion slices. Journal of Food Engineering, 71(3), pp: 282-286. 2005.

Recibido: $14 / 03 / 2019$.

Aprobado: 22/07/2019. 